\title{
Microbristle in gels: Toward all-polymer reconfigurable hybrid surfaces
}

\section{Citation}

Kim, Philseok, Lauren D. Zarzar, Xuanhe Zhao, Alexander Sidorenko, and Joanna Aizenberg. 2010. "Microbristle in Gels: Toward All-Polymer Reconfigurable Hybrid Surfaces." Soft Matter 6 (4): 750. doi:10.1039/b920392c.

\section{Published Version}

doi:10.1039/b920392c

\section{Permanent link}

http://nrs.harvard.edu/urn-3:HUL.InstRepos:34609600

\section{Terms of Use}

This article was downloaded from Harvard University's DASH repository, and is made available under the terms and conditions applicable to Open Access Policy Articles, as set forth at http:// nrs.harvard.edu/urn-3:HUL.InstRepos:dash.current.terms-of-use\#OAP

\section{Share Your Story}

The Harvard community has made this article openly available.

Please share how this access benefits you. Submit a story.

Accessibility 


\title{
Microbristle in Gels: Toward All-Polymer Reconfigurable Hybrid Surfaces
}

\author{
Philseok Kim ${ }^{1,2}$, Lauren D. Zarzar ${ }^{3}$, Xuanhe Zhao ${ }^{1}$, Alexander Sidorenko ${ }^{4}$, Joanna
} Aizenberg*1,2,3

1. School of Engineering and Applied Sciences, Harvard University, Cambridge, MA

2. Wyss Institute for Biologically Inspired Engineering, Harvard University, Cambridge, MA

3. Department of Chemistry and Chemical Biology, Harvard University, Cambridge, MA

4. Department of Chemistry and Biochemistry, University of the Sciences in Philadelphia, Philadelphia, PA

Corresponding author: Prof. Joanna Aizenberg, e-mail: jaiz@seas.harvard.edu

\section{$\underline{\text { Abstract }}$}

We report on the fabrication of biologically-inspired "smart" surfaces using hybrid architectures comprising polymer microbristle embedded in a hydrogel layer. The dynamic bending of the microposts - the passive structural element in the design - and their return to the upright orientation are achieved during the volume-phase transition of the hydrogel layer-the active element of the structure - upon hydration/dehydration. We compare the performance of the hybrid architectures bearing soft and stiff microposts and show that the use of soft polymeric materials results in bending actuation of the posts in cases where actuation of identically-sized posts of stiffer materials, such as silicon, would not have been possible. Modeling of the actuation process and the supporting experimental results confirm that the bending orientation of the microposts can be individually controlled by modulating the thickness gradients in the active hydrogel layer achieved by transferring micropatterns to the liquid-phase hydrogel precursor. Such procedures orchestrate coordinated actuation of the microbristle and make it possible to create elaborate reconfigurable micropatterns, such as opening/closing microflorets and microtraps. In combination with diverse hydrogel systems exhibiting response to various stimuli, these "smart" hybrid all-polymer architectures open a new avenue in advanced functional materials that harness the adaptive nature of these structures for various applications. 


\section{INTRODUCTION}

Responsive, smart systems with dynamically and reversibly switching surface patterns and surface properties at various length scales are gaining interest in microactuation systems, sensors, microfluidics, biomedicine, tissue engineering, biomimetics, robotics, architecture, optics, and photonics. ${ }^{1-9}$ Combined with responsiveness and the ability to pattern such materials, actuators with continuously tunable and controllable direction of actuation can allow the fabrication of complex surface patterns relevant to important applications such as switching of wetting behavior, generation of flow, propulsion and transportation, controlled cell attachment/detachment, capture/release systems, energy conversion, and color mimicking. ${ }^{6-8,10-14}$ Nature utilizes smart and responsive actuation systems for a variety of functions; for example, actuation of claw-like pedicellaria in echinoderms is believed to provide anti-fouling and defense functions, hygroscopic wheat awns facilitate seeding in the ground, flagella and cilia generate propulsion and locomotion, Venus flytraps open and close to catch prey, movements of the pigment-filled sacks in squids result in dynamic color changes, etc. ${ }^{15-21}$ Typically, these bioactuation systems are based on soft structures, which comprise both the active and passive parts of the actuator.

Recently, we have reported reversible actuation of surface structures by combining a rigid (inorganic), passive structural element (silicon nanobristles) with a soft (organic), active layer (humidity-sensitive hydrogel), with which dynamic switching of wetting properties and various actuated micropatterns were achieved. ${ }^{8,10}$ We have shown that it is critical for these passive Si nanostructures to have a very high aspect ratio in order to ensure the bending of the 
stiff Si bristle by the forces generated in the contracting hydrogel. Moreover, the strain at the base often exceeds the fracture strain of silicon and results in breaking of the Si posts upon contraction of the hydrogel. ${ }^{8}$ This problem makes the fabrication process of the Si nanobristle highly expensive and sets significant limitations to the practical application of these structures. The fabrication of micron-sized Si features with reduced aspect ratios is significantly simplified, but the flexural modulus of these structures will drastically increase and the forces generated in the contracting hydrogel would not be sufficient to actuate such stiff elements. Polymeric materials, on the other hand, perfectly match the required bending conditions for microstructures that can be easily made to actuate in response to the reversible volume changes in the supporting hydrogel layer. Polymers can also be tailored for various mechanical, chemical, and surface properties as well as for responsiveness to humidity, $\mathrm{pH}$, temperature, light, molecular and mechanical stimuli, and electric field. ${ }^{2-4,14,22}$ As in biological systems, the polymeric structures can be therefore designed to play a role of both the passive and the active elements in the actuatable hybrid bristle arrays. In an effort to utilize these advantages of soft materials, a simple method to create replicas of silicon "master" structures has been reported by our group; a negative replica of the silicon structure is molded by polydimethylsiloxane (PDMS) and backfilled with liquid pre-polymers, which are then solidified by curing and removed from the PDMS mold to yield a replica. ${ }^{23}$ This method provides greater flexibility in the choice of materials with tunable mechanical properties as well as chemical and biological functionalities. The use of soft materials will make possible the fabrication of complex patterns with much less effort. In addition, lower forces are required to bend the soft microposts (even with increased geometrical stiffness, e.g. at a lower aspect ratio) due to their reduced material stiffness (2-3 orders of magnitude reduction of the flexural modulus compared to Si) using volume-phase transitions of 
hydrogel. Finally, the elastic nature of the polymeric material should eliminate the problem associated with the fracture of the Si posts and facilitate the restoration of the original shape when strain is removed during hydrogel swelling.

In this paper, we report on the fabrication of such all-polymeric, hybrid, responsive soft surfaces based on the combination of passive polymeric micro-structures embedded in an active hydrogel layer. We demonstrate the reversible switching of surface structures into a variety of non-trivial patterns at the micrometer-scale induced by the volume changes in the hydrogel upon hydration/drying. We also report a finite element simulation and the supporting experimental results showing how the directionality of the actuation can be manipulated by controlled deposition of the hydrogel layer using pre-patterned surfaces. These results will provide insights into the understanding of the dynamic actuation behavior of hybrid actuator systems and offer the basis for the rational design of dynamic surfaces.

\section{RESULTS AND DISCUSSION}

\section{Synthesis and fabrication of the dynamic surfaces.}

The fabrication procedure for the reconfigurable soft hybrid surface is shown in Figure 1. As a soft passive element in the actuator system, a square array of microposts having a circular cross-section (pitch $=8 \mu \mathrm{m}$, height $=10 \mu \mathrm{m}$, post diameter $=1.5 \mu \mathrm{m})$ was used (Figure $1 \mathrm{~d})$. This soft bristle was replicated from a "master" Si microstructure using PDMS molding technique. ${ }^{23}$ The polymer used for the replication was a mixture of commercial one-component photo-curable epoxy resin (UVO114, Epotek) and bifunctional monomer, glycidylmethacrylate (GMA, Aldrich). GMA has both a glycidyl and a methacrylate group. The former reacts with the epoxy resin during the photo-curing (Figure 1a), and the latter allows 'grafting-through' polymerization 
of acrylamide-based hydrogel on the surface of the polymer microstructures (Figure 1b). ${ }^{10}$ The flexural modulus of the resulting polymer (GMA-modified UVO114) determined using fourpoint flexure experiments was found to be $1.5 \mathrm{GPa}$. The surface of the replicated epoxy microposts was superhydrophobic (water contact angle $=158.0 \pm 1.1^{\circ}$ ) and the metastable Cassie state of a water droplet persisted (see Figure 1d, inset). UVO114 with no GMA was used to replicate the relief structure of a confining patterned surface (Figure 1c). As a soft, responsive, and active 'muscle' part in the actuator system, an aqueous solution of either thermally or UV cross-linkable acrylamide (AAm) hydrogel with a fixed cross-linking density was used (Figure 1b). The hydrogel precursor solution was applied to the polymer micropost substrate. Ethanol was used to improve the wetting of the superhydrophobic substrates by the aqueous hydrogel solution. A confining surface was then placed to cover the hydrogel solution. The hydrogel was formed by using either thermosensitive $\left(45^{\circ} \mathrm{C}, 1\right.$ hour $)$ or photosensitive $\left(c a .30 \mathrm{~mJ} / \mathrm{cm}^{2}\right.$ at 276 $\mathrm{nm}$ ) initiators. The confining surface was removed after swelling of the hydrogel layer in deionized water to reveal the micropost/hydrogel hybrid surface.

\section{Analysis and modeling of the actuation process.}

Upon dehydration, the bending of the microposts occurs due to the strain from the shrinking hydrogel. The bending is accompanied by a gradual increase of the elastic energy stored in the post. When hydrogel swells, the stored elastic energy is released returning the posts to their unstrained upright configuration. Analogous to the spring constant of an elastic material, the effective stiffness $\left(S_{\text {eff }}\right)$ of a post is an important parameter in comparing the difference in geometry and modulus of the materials. For two different posts with a circular cross-section, the ratio of their effective stiffness can be expressed as: 


$$
\frac{S_{1 \mathrm{eff}}}{S_{2_{\mathrm{eff}}}}=\left(\frac{E_{1}}{E_{2}}\right)\left(\frac{l_{2}}{l_{1}}\right)^{3}\left(\frac{d_{1}}{d_{2}}\right)^{4}
$$

where $E$ is the bending modulus, $l$ is the length, and $d$ is the diameter of the posts, respectively. ${ }^{23}$ In our previous study, the Si posts were required to have sub-micrometer-scale dimensions $(\mathrm{d} \leq$ $300 \mathrm{~nm})$ and a high aspect ratio $(\mathrm{AR}=l / d>20)$ for significant bending to occur without breaking, due to the high stiffness of silicon $(E=200 \mathrm{GPa}){ }^{8}$ Therefore, if a soft material having a 2-3 orders of magnitude lower modulus is used for the construction of the bristle, geometrically stiffer posts (e.g. those having a low aspect ratio and micrometer-scale dimensions) can be bent by applying the same hydrogel muscle. The fabrication of an arbitrary array of posts in this dimension range is substantially simplified, and a more robust actuation system showing no fracture of the structural elements results. In particular, an exemplary epoxy microbristle $(d=$ $1.5 \mu \mathrm{m}, l=10 \mu \mathrm{m}, \mathrm{AR}=6.6, E=1.5 \mathrm{GPa}$ ) used in this study (Figure 1d) would have similar effective stiffness $\left(S_{\text {eff }}\right)$ as the Si nanobristle $(d=0.3 \mu \mathrm{m}, l=6 \mu \mathrm{m}, \mathrm{AR}=20, E=200 \mathrm{GPa})$ described in our previous study. ${ }^{8}$ Thus, the use of soft polymeric materials will allow the bending of the posts having a much larger range of feature sizes, and geometrically stiffer structures (e.g. low aspect ratio microposts, microblades) can be actuated, while the actuation of such Si structures would be impossible.

Another important feature of the design of a "smart" actuation system is the ability to induce a desired patterned actuation. Such property relies on a precise control of the direction of bending for each individual post in the hybrid system. We hypothesized that the bending direction can be governed by the local changes in the height profile of the hydrogel layer. The effect of the hydrogel profile on the directional actuation was examined by using a model shown 
in Figure 2. A numerical model was constructed for a soft micropost-hydrogel system to visualize the expected bending motion of the posts, when the hydrogel layer that undergoes volume-phase transition is grown with a continuously changing height profile (Figure 2). The model was implemented in the finite-element package, ABAQUS, with a user-defined subroutine, UHYPER, that describes the swelling/de-swelling behavior of the hydrogel. ${ }^{24}$ The geometry and properties of the polymer microposts were taken as values given above (circular microposts, $d=$ $1.5 \mu \mathrm{m}, l=10 \mu \mathrm{m}, p($ pitch $)=8 \mu \mathrm{m}, E=1.5 \mathrm{GPa})$ and the swelling ratio of the hydrogel was set to 5 for the simulation. In order to simplify the calculation, a plane strain boundary was assumed for the model. When the hydrogel is in a wet state (Figure 2a), the polymer microposts stand upright. As the hydrogel is drying (Figure 2b-d), the microposts bend toward the regions with thicker gel layers.

Ideally, for an unpatterned, uniform hydrogel film which is grown isotropically around each post, only abrupt bending by buckling can happen and continuous bending of the post, as modeled in Figure 2, cannot occur as the sum of the deflection forces becomes zero. However, in reality, even with the unpatterned hydrogel layer, the continuous bending of the posts would occur, since any imperfection in the microstructure would result in a non-zero net deflection force. Thus, one would expect random bending orientation of microposts in such systems. This analysis implies that by controlling the local thickness gradients in the hydrogel layer, one can induce the directional motions of the embedded polymeric microstructures into reversible patterns.

\section{Experimental examples of smart, reconfigurable surfaces.}

The validity of the theoretical considerations and modeling presented above was demonstrated in the following exemplary systems. Figure 3 shows the responsiveness of the 
micropost/hydrogel hybrid soft surface under wet or dry conditions. The bending of the microposts was clearly observed with the reduction of the hydration level, and the restoration of the bent microposts back to the straight, upright state occurred upon rehydration. This change was reversible and the actuated patterns were dynamically switched by repeated cycling of exposure to water and drying (see Supporting Information for actuation movies). Moreover, the hydrogel layer showed a strong adhesion to the substrates prepared from GMA-modified UVO114 and remained on the micropost substrate after many actuation cycles, while it delaminated easily from the substrates prepared using only UVO114. This confirmed the covalent attachment of the hydrogel to the micropost substrates. Therefore, the results with GMA-modified UVO114 suggest that a broad range of soft materials can be used as the passive segment in our actuation system when a functional group that can couple with the acrylate-based hydrogel is present. In fact, similar results were obtained by using commercially available acrylate-based resin (OG603, Epotek) and GMA-modified SU-8 resist (Microchem). The response time of the actuation was found to depend on the hydrogel thickness (typically a few micrometers) due to the variation in the diffusion length of water molecules through the hydrogel layer.

The hydrogel profile in our experiments was controlled by using an appropriately designed confining surface (Figure 1c). Figure 3a illustrates the actuation in the hybrid structure prepared using a flat confining surface. Interestingly, we observed that the bending orientation was not purely stochastic as should be expected in such a system, but it showed rather uniform, largely one-directional actuation affected by the experimental conditions. Generally in the case of a flat hydrogel layer, the bent microposts formed large domains, each with a different but uniform bending orientation. Once a post bends in a particular direction, it produces a local 
anisotropy in the thickness of the hydrogel, thus biasing the neighboring posts to bend in the same direction. As a result, a bending post at a defect site can effectively induce the propagation of the same bending orientation in a large area forming domains with uniform (though uncontrolled) directed actuation. Controlled, one-directional actuation across the entire system can be achieved by introducing specific asymmetry into the hybrid design confined by the flat surface. For example, the peeling orientation during replication imposes a small tilt onto the replicated polymeric structures, leading to a non-zero deflection force acting directionally on each post and resulting in a specific, one-directional actuation. Similarly, tilting of the substrate or introducing a wedged gap between the micropost and the confining surface would create a thickness gradient in the gel and, thereby, directional bending of the posts toward the thicker gel. $^{25}$

The capability to reconfigure the surfaces into more complex switchable patterns at a microscale is a very attractive and application-relevant feature of the future dynamic, "smart" materials. ${ }^{2}$ Here we show that patterning of hydrogel layers around each micropost and/or a section of microposts with local thickness gradients (Figure 2) can lead to coordinated bending of the microposts to form arbitrary surface patterns. Hydrogel patterning was achieved by direct imprinting method, in which the growth of the hydrogel layer on the micropost substrate was confined in space by using another micropatterned substrate, as described in Figure 1. A variety of unique reconfigurable surfaces with complex, dynamic patterns were produced (see Figure 3b, c and Supporting Information), and all of these micropatterns exhibited reversible switching between dry (bent, patterned) and wet (straight, original square array) configurations. For example, dynamic microtrap crosses wrapped in a hydrogel were formed when a confining surface was patterned with a commensurate micropost array (Figure 3b). When the hydrogel 
growth was confined with a honeycomb pattern (wall-to-wall distance $=35 \mu \mathrm{m}$, depth of a honeycomb well $=10 \mu \mathrm{m}$ ) over a square array of microposts, an array of blooming microflorets was formed (Figure 3c).

Interestingly, in the latter case three different actuation types can be induced. Opening microflorets (posts bending outward from the centers of the hexagonal wells upon drying) were obtained with an untreated honeycomb microwell confinement surface (Figure 4a), while closing microflorets (posts bending inward toward the center of the hexagonal wells upon drying) were obtained with an oxygen plasma-treated confinement surface (Figure $4 b$ ). We attribute the observed difference in the actuation direction to the differences in the wetting behavior of the aqueous hydrogel precursor solution at the walls of the honeycomb pattern. The honeycombpatterned substrate made from unmodified UVO114 is hydrophobic (water contact angle $=136.5$ $\left.\pm 1.4^{\circ}\right)$. Oxygen plasma treatment $(100 \mathrm{~W}, 20 \mathrm{sec})$ renders this surface hydrophilic (water contact angle $=59.8 \pm 7.1^{\circ}$ ). When the aqueous hydrogel precursor solution is confined between the micropost substrate and the honeycomb microwell-patterned substrate, the solution can be in contact either with only the walls of the honeycomb (untreated, non-wetting, Cassie state) or with the inner surface of the honeycomb (oxygen plasma-treated, wetting, Wenzel state). The experimental observation matches well with the modeling results presented in Figure 2; that is, when the thicker hydrogel corresponds to the contact with the walls of a honeycomb, an outward bending toward the thicker gel at the wall of the well is expected, which will be manifested as opening florets; when the thicker hydrogel regions form inside the honeycomb microwells due to wetting, an inward bending of the posts will be expected, which will be manifested as closing florets (Figure 4, top). Atomic force microscopy (AFM) images revealed how the profile of the 
resultant hydrogel layer is changed by controlling the wetting property of the confining surface (Figure 4, bottom).

When the wetting of the confining surface is between these two extreme examples or a thicker hydrogel layer is created, the resultant height variations in the hydrogel are diminished and microflorets with unidirectionally bending microposts can be obtained (Figure 3c). The orientation of bending of the microposts in this case is rather defined by large scale imperfections, such as wedging, tilting, or curvature in the substrate. These imperfections give rise to the variations in the thickness of the hydrogel layer, which determine the directional movement of the microposts, while the overall honeycomb pattern is still imposed by the profile of the confining surface. We envisage that the controlled incorporation of defects in a systematic way to create significant local perturbations can lead to the fabrication of even more elaborate reconfigurable surfaces. Figure 5 illustrates the latter statement by presenting an experiment in which honeycomb confinement was combined with an accidentally created chiral defect. Twisting microflorets with gradually rotating orientations of the microposts defined by the defect were obtained, resembling the motion of a camera iris (See Supporting Information for the actuation movie).

It is noteworthy that the structural reconfiguration of the hybrid surfaces and the associated changes in the thickness of the hydrogel induce color changes during the actuation (see Figure 5). Thus, these new actuation systems can be used to create "smart" surfaces and coatings that dynamically change color and transmission or reflection of light in response to environmental conditions in a manner similar to the camouflaging animals and insects. We also believe that the closing/opening reconfiguration of microflorets (Figure 4) and microtraps (Figure 3b) can be potentially utilized to capture and release particles. ${ }^{7}$ Moreover, the 
coordinated motion of the microstructures could be applied to induce directional propulsion of particulate matter. $^{26}$

\section{CONCLUSIONS AND OUTLOOK}

We have demonstrated the fabrication of adaptively switching surface patterns by using a hybrid structure composed of an array of passive polymeric microposts put in motion by an active hydrogel layer. This all-polymeric actuation system offers an opportunity to utilize a variety of polymeric materials. The use of soft materials allowed the bending actuation to occur in a much broader range of sizes and mechanical properties of microbristle that is impossible with the organic/inorganic hybrids bearing stiff elements of same geometry. Simple microimprinting patterning method afforded the precise manipulation of the direction of the actuation in accordance with simulation that predicted the movement of the posts embedded in a gel with a non-uniform thickness. This approach offers a unique, versatile technique to generate soft, responsive, reconfigurable hybrid surfaces with complex micropatterns. Our method, with the combinations of different geometric elements and various active soft materials (e.g. responsive to $\mathrm{pH}$, temperature, and electrical stimuli), opens a door to a broad materials choice for soft actuators with versatile mechanical and chemical properties leading toward the fabrication of adaptable soft architectures useful for various applications.

\section{EXPERIMENTAL}

Acrylamide (AAm), N,N'-methylenebisacrylamide (bis-AAm), ammonium persulfate (APS), glycidyl methacrylate (GMA), and (tridecafluoro-1,1,2,2-tetrahydrooctyl) triethoxy silane 
were purchased from Sigma-Aldrich (St. Louis, MO). Polydimethylsiloxane (PDMS) (DowSylgard 184) was purchased from Ellsworth (Germantown, WI) and UVO-114 was purchased from Epoxy Technology (Billercia, MA). All the chemicals were used as received.

Silicon masters of the post and honeycomb arrays were fabricated using the Bosch process as described elsewhere. ${ }^{27,} 28$ Silicon masters were then fluorosilanized with (tridecafluoro-1,1,2,2-tetrahydrooctyl)triethoxy silane in a desiccator under vacuum for at least 24 hours to reduce sticking of the silicon to the PDMS.

PDMS of a 10:1 ratio of prepolymer-to-curing agent was prepared by mixing for 3 minutes and bubbles were removed under vacuum. To create negative replicas, the PDMS mixture was poured over the silanized silicon master in a petri dish, put under vacuum again to remove residual bubbles, and then cured for 2 hours at $70^{\circ} \mathrm{C}$. After cooling, the PDMS was gently pulled off of the silicon masters.

To make a polymer replica, a 9:1 mixture of UVO-114 and GMA was poured over the PDMS mold, and a glass slide was placed over the epoxy resin to serve as a flat backing, and spacers of $500 \mu \mathrm{m}$ thickness were used to yield an area of polymer with a coverage of about 1 $\mathrm{cm}^{2}$. The epoxy resin mixture was cured under a UV lamp $(100 \mathrm{~W}$, Black-Ray with a $385 \mathrm{~nm}$ band-pass filter, power at $385 \mathrm{~nm}: \sim 10 \mathrm{~mW} / \mathrm{cm}^{2}$ ) for about 20 minutes and subsequently peeled off of the PDMS mold to yield an array of polymer posts that were an exact replica of the original silicon master. The same replication procedure was followed for the confining surfaces except that $100 \%$ UVO-114 was used. A $100 \mathrm{~W}$ plasma cleaner (Model Femto, Diener GmbH, Nagold, Germany) was used for oxygen plasma treatment of the confining surface for 20 seconds if necessary. 
Hydrogel precursor solution (40\% AAm, 2\% bis-AAm, 2\% APS by weight in deionized water) was placed on the micropost surface and then the confining layer was placed face down over the posts and held clamped together by binder clips. The hydrogel was cured in an oven at $45^{\circ} \mathrm{C}$ for 90 minutes and then the sample was placed in deionized water at room temperature for 45 minutes to swell the hydrogel before unclipping the slides and separating the post and confining surface. Samples were successively moistened with a wet tissue and dried with compressed air to change the hydrogel's contraction level for the demonstration of actuation.

Optical imaging and video recording was done on an Olympus IX71 inverted microscope using StreamPix v.3 software and QImaging EXi Blue and Evolution VF cameras. Samples were sputter coated with Pt/Pd for imaging in the Zeiss Ultra55 FESEM. AFM images were taken with Veeco NanoMan AFM using Si probes in TappingMode under ambient conditions. Four-point flexure experiments were performed using a dual column universal materials testing system (Model 5566, Instron) with ca. $63 \mathrm{~mm}$ long bar specimens with a bending rate of 50 $\mathrm{mm} / \mathrm{min}$ until a maximum of $4 \mathrm{~mm}$ deflection or $500 \mathrm{~N}$ applied force. The load force-deflection curve was linearly fitted to find the slope which was subsequently used to find the Young's modulus of the polymers tested. ${ }^{29}$

\section{NOTES AND REFERENCES}

This project was partially supported by the MRSEC program of the National Science Foundation under award number DMR-082048 and by the WYSS Institute for Biologically Inspired Engineering at Harvard University. The work was performed in part at the Center for Nanoscale Systems (CNS), a member of the National Nanotechnology Infrastructure Network (NNIN), 
which is supported by the National Science Foundation under NSF award number ECS- 0335765 .

We thank Alexander E. Epstein for the help in the four-point flexure test.

1. S. Minko, Responsive Polymer Materials: Design and Applications, Blackwell, Ames, IA, 2006 and references therein.

2. D. Kuckling, Colloid and Polymer Science, 2009, 287, 881-891.

3. Y. Osada and D. E. D. Rossi, eds., Polymer Sensors and Actuators, Springer-Verlag, Berlin, Germany, 2000.

4. Y. Bar-Cohen, ed., Electroactive Polymer (EAP) Actuators as Artificial Muscles: Reality, Potential, and Challenges, 2nd edn., SPIE, Bellingham, WA, USA, 2004.

5. Z.-g. Zhou and Z.-w. Liu, Journal of Bionic Engineering, 2008, 5, 358-365.

6. V. V. Yashin and A. C. Balazs, Science, 2006, 314, 798-801.

7. B. Pokroy, S. H. Kang, L. Mahadevan and J. Aizenberg, Science, 2009, 323, 237-240.

8. A. Sidorenko, T. Krupenkin, A. Taylor, P. Fratzl and J. Aizenberg, Science, 2007, 315, 487-490.

9. K. Jung, J. C. Koo, J.-d. Nam, Y. K. Lee and H. R. Choi, Bioinspiration and Biomimetics, 2007, 2, S42-S49.

10. A. Sidorenko, T. Krupenkin and J. Aizenberg, Journal of Materials Chemistry, 2008, 18, 3841-3846.

11. J. d. Toonder, F. Bos, D. Broer, L. Filippini, M. Gillies, J. d. Goede, T. Mol, M. Reijme, W. Talen, H. Wilderbeek, V. Khatavkar and P. Anderson, Lab on a Chip, 2008, 8, 533541.

12. B. A. Evans, A. R. Shields, R. L. Carroll, S. Washburn, R. R. Falvo and R. Superfine, Nano Letters, 2007, 7, 1428-1434.

13. M. T. Yang, N. J. Sniadecki and C. S. Chen, Advanced Materials, 2007, 19, 3119-3123.

14. V. Kozlovskaya, E. Kharlampieva, B. P. Khanal, P. Manna, E. R. Zubarev and V. V. Tsukruk, Chemistry of Materials, 2008, 20, 7474-7485.

15. J. D. McKenzie and I. V. Grigolava, Biofouling, 1996, 10, 261-272.

16. R. Elbaum, L. Zaltzman, I. Burgert and P. Fratzl, Science, 2007, 316, 884-886. 
17. B. Bhushan, Philosophical Transactions of The Royal Society A, 2009, 367, 1445-1486.

18. I. Burgert and P. Fratzl, Philosophical Transactions of The Royal Society A, 2009, 367, 1541-1557.

19. R. M. Kramer, W. J. Crookes-Goodson and R. R. Naik, Nature Materials, 2007, 6, 533538.

20. B. Mitchell, R. Jacobs, J. Li, S. Chien and C. Kintner, Nature, 2007, 447, 97-102.

21. Y. 1. Forterre, J. M. Skotheim, J. Dumais and L. Mahadevan, Nature, 2005, 433, 421-425.

22. C. L. v. Oosten, C. W. M. Bastiaansen and D. J. Broer, Nature Materials, 2009, 8, 677682.

23. B. Pokroy, A. K. Epstein, M. C. M. Persson-Gulda and J. Aizenberg, Advanced Materials, 2009, 21, 463-469.

24. W. Hong, Z. Liu and Z. Suo, International Journal of Solids and Structures, 2009, 46, 3282-3289.

25. D. Wu, Q.-D. Chen, B.-B. Xu, J. Jiao, Y. Xu, H. Xia and H.-B. Sun, Applied Physics Letters, 2009, 95, 091902.

26. Y. Murase, S. Maeda, S. Hashimoto and R. Yoshida, Langmuir, 2009, 25, 483-489.

27. T. N. Krupenkin, J. A. Taylor, T. M. Schneider and S. Yang, Langmuir, 2004, 20, 38243827.

28. S. A. McAuley, H. Ashraf, L. Atabo, A. Chambers, S. Hall, J. Hopkins and G. Nicholls, Journal of Physics D: Applied Physics, 2001, 34, 2769-2774.

29. A. R. Ragab and S. E. A. Bayoumi, Engineering Solid Mechanics: Fundamentals and Applications, CRC Press, Boca Raton, FL, 1998. 
(a)

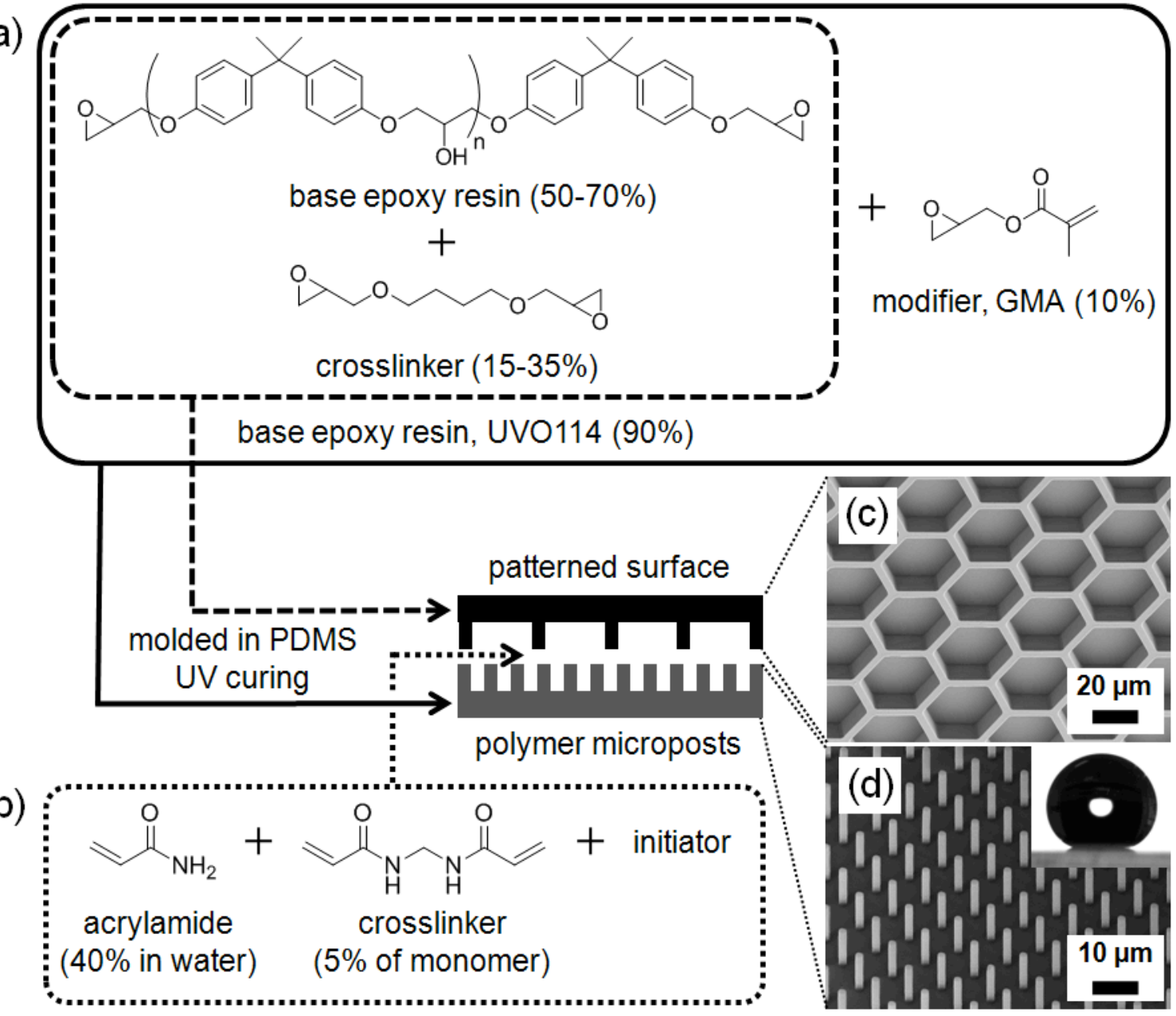

Figure 1. Schematics of the fabrication of all-polymer reconfigurable surfaces. (a): Synthetic scheme for the preparation of the polymer microposts and confining surfaces, (b): Synthetic scheme for the preparation of the hydrogel, (c): Scanning electron microscope (SEM) image of an exemplary confining surface bearing a honeycomb pattern, (d): SEM image of polymer microposts. Inset shows a water droplet on the superhydrophobic polymer micropost substrate. 
(a)

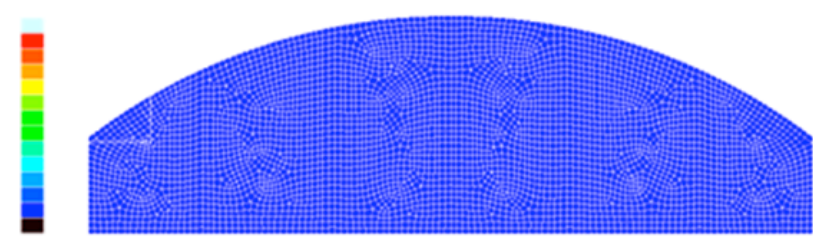

(c)

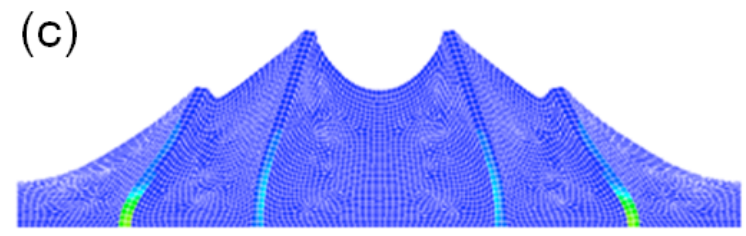

(b)

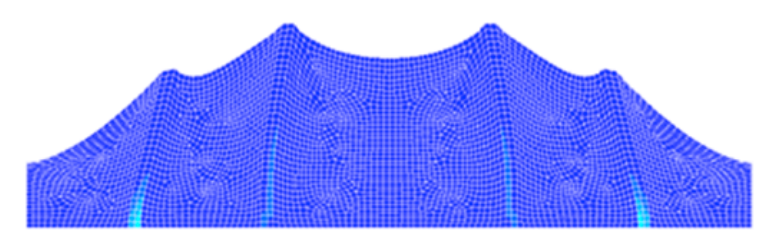

(d)

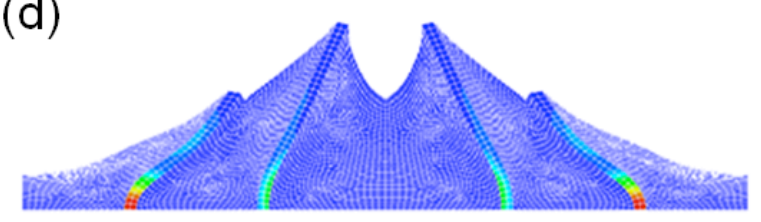

Figure 2. 2D-finite element simulation results, showing the deformation of soft microposts embedded in a hydrogel layer with varying thickness. As the hydrogel layer dries (from (a) to (d)), the microposts are bending toward the area with a thicker hydrogel. The strain is represented as a rainbow scale in each micropost where red - orange areas indicate a larger strain than blue - purple areas. 


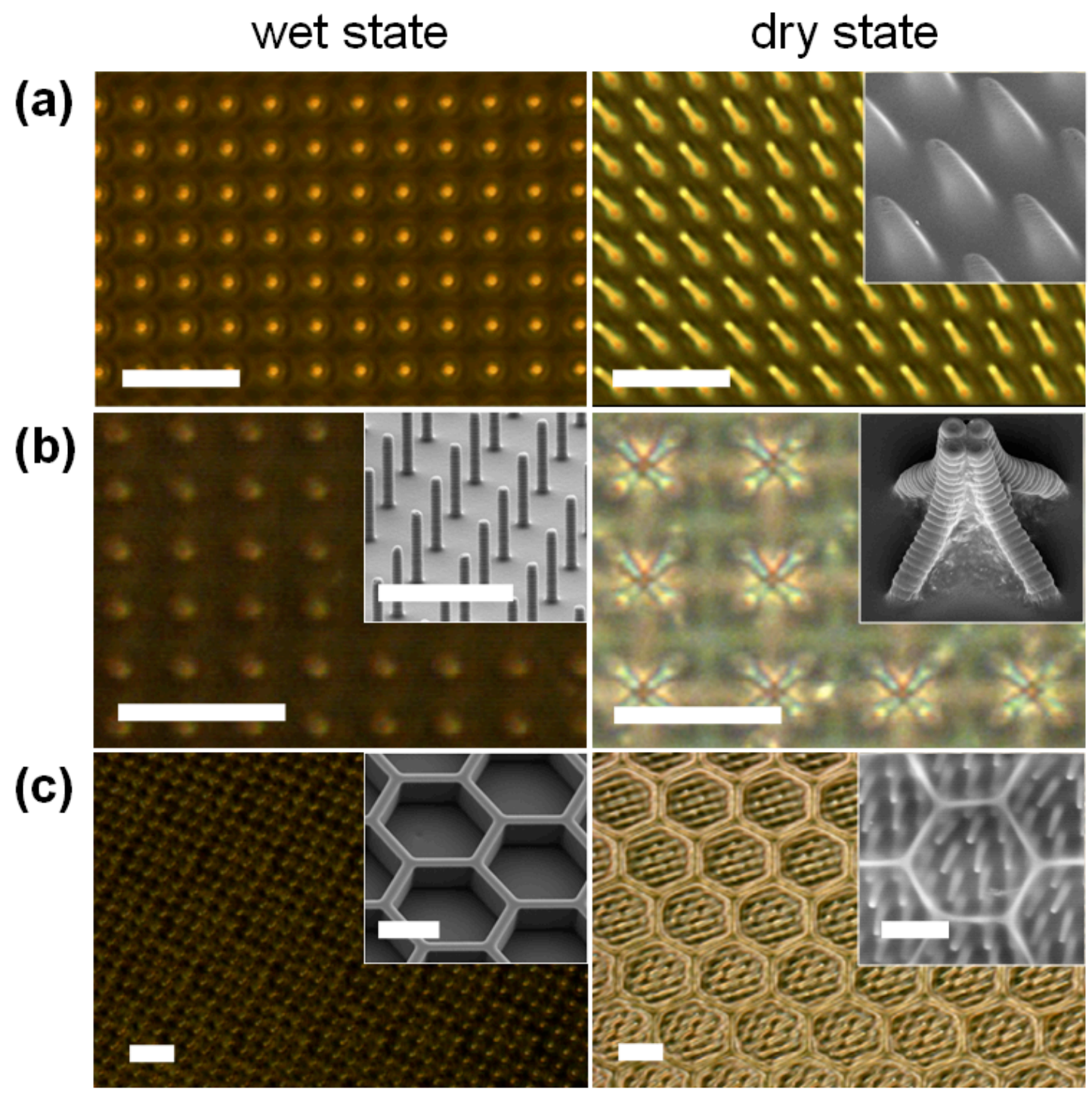

Figure 3. Optical microscope images of wet (left) and dry (right) states of reconfigurable polymeric micropost/hydrogel hybrid surfaces prepared by using various confinement geometries. Inset images are SEM micrographs of the confining surface patterns (left) and of the polymer micropost-hydrogel hybrid structures (right). (a): Flat confining surface, (b): Confining surface bearing an array of microposts, (c) Confining surface bearing honeycomb microwells. Scale bars are $20 \mu \mathrm{m}$. See Supporting Information for actuation movies. 
(a) without $\mathrm{O}_{2}$ plasma treatment

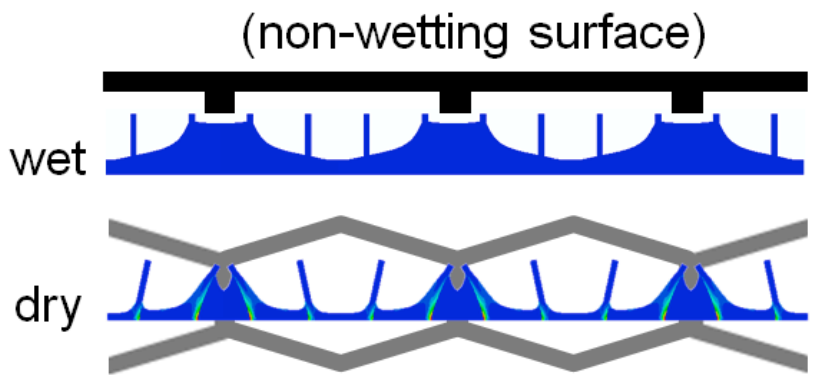

opening microflorets

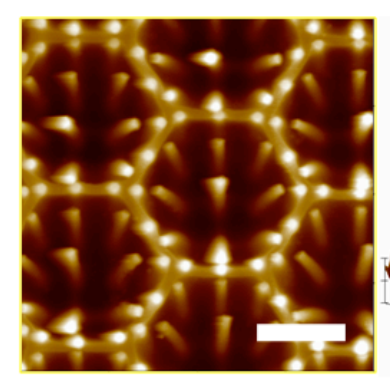

(b) with $\mathrm{O}_{2}$ plasma treatment (wetting surface)
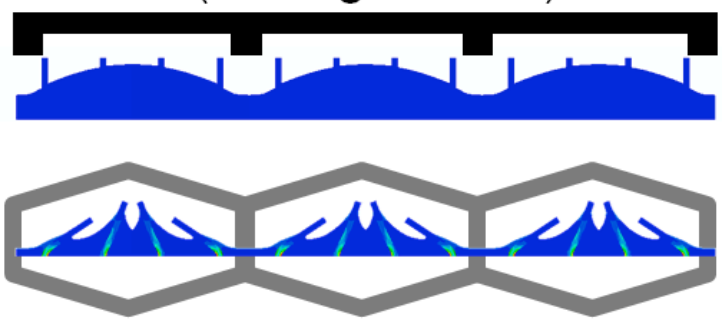

closing microflorets

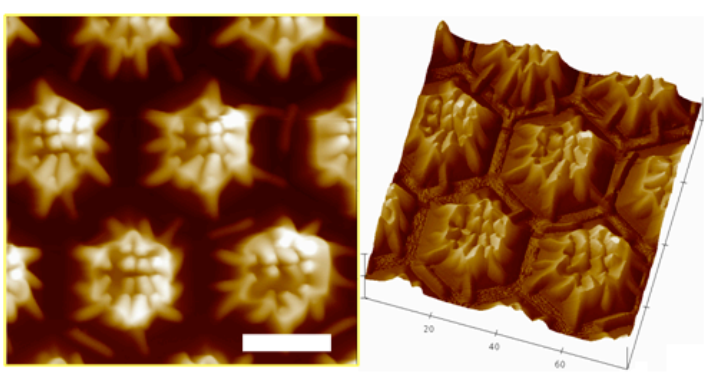

Figure 4. Comparison of finite element simulation and the experimental observations for the formation of opening (a) and closing (b) microflorets. Top: simulation results comparing the patterns that originate from confining surfaces with different wetting properties. Bottom: Atomic force microscope (AFM) height images (top view and 3D view) of microflorets exhibiting opening and closing actuation upon dehydration. Scale bars are $20 \mu \mathrm{m}$. 


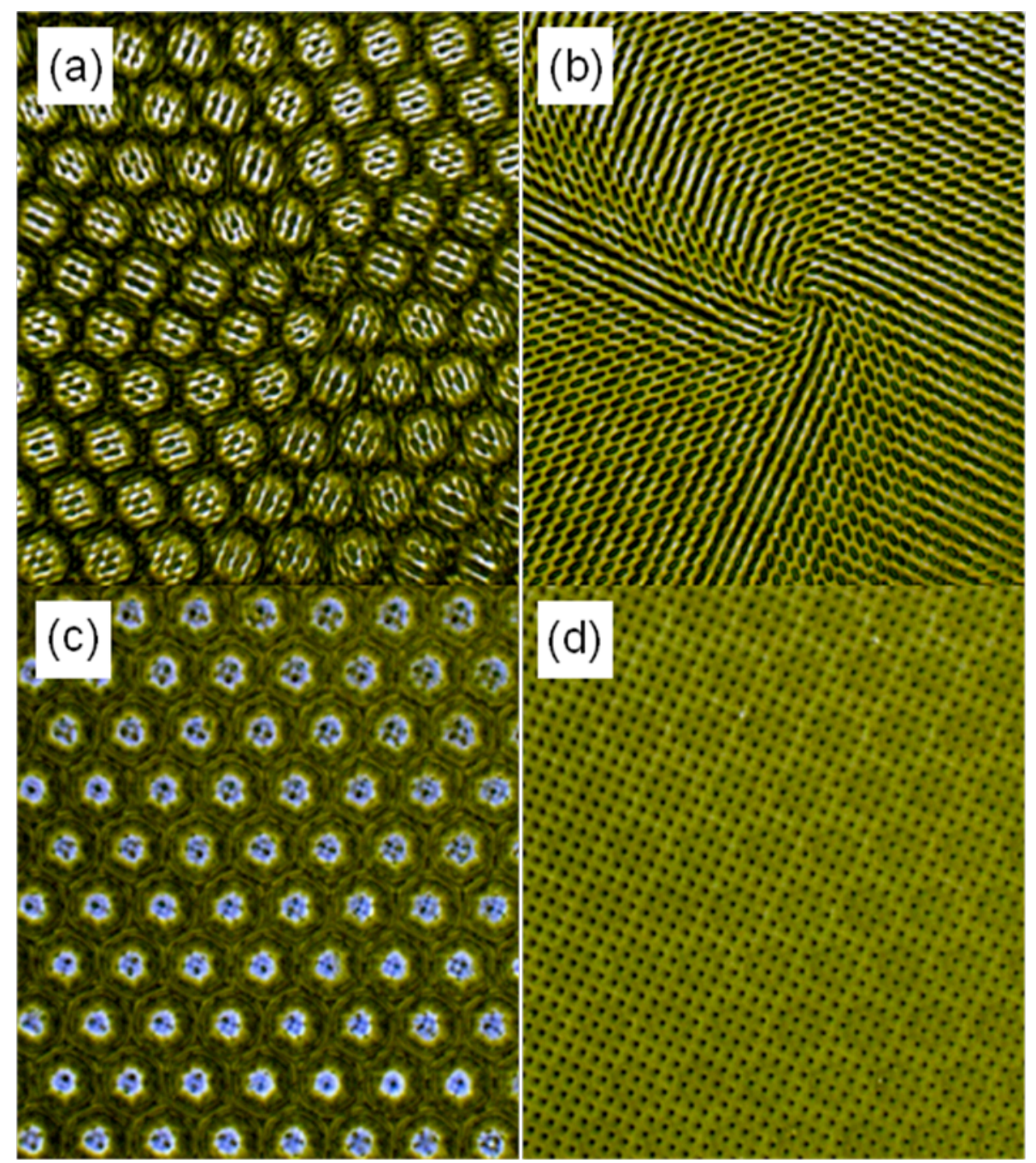

Figure 5. Complex, spiral actuation of the posts in the microflorets pattern induced by a chiral defect. (a): Dry state, (b) Dry to wet transition, (c) Wet state, (d) Wet to dry transition. Note a clear color change during the actuation. See Supporting Information for actuation movies. 


\section{Supporting Information}

Video clips:

movie1-modeling of actuation.avi (2.5 MB) - full movie of the modeling result described in Figure 2.

movie2-microtrap actuation.avi (2.1 MB) - actuation movie of Figure 3b.

movie3-microfloret actuation3.avi (3.8 MB) - actuation movie of Figure 3c.

movie4-closing microfloret actuation.avi (1.7 MB) - actuation movie of closing microflorets depicted in Figure 4b.

movie5-microfloret with a defect.avi (5.5 MB) - actuation movie of Figure 5. 